of such a degree of hydrocephalus that there seems to be scarcely any brain substance left.

In many cases of tuberculous meningitis streptomycin seems to maintain the condition of the patient when treatment was commenced, but little subsequent improvement is seen. Such experiences support the case for the inclusion of some adjuvant antibacterial agent with the streptomycin, in the hope that a synergistic action such as is seen with sulphonamide and penicillin might be found.

Finally, it must be realized that the streptomycin treatment of tuberculous meningitis cannot be lightly undertaken. Apart from full laboratory facilities for controlling the clinical pathology of a case much extra nursing is required, continuous medical supervision is necessary, and from the patient's point of view there will be a course of intramuscular injections lasting for six months or more, apart from intrathecal and general supporting treatment. Only the fullest and continuing co-operation of everybody concerned can give streptomycin treatment the fair trial it deserves, and even in the most successful cases with apparent cure, prolonged subsequent observation is necessary owing to the possibility of relapse.

\section{AdDendum}

These observations were made at the Hospital for Sick Children, which was one of the centres taking part in the investigation by the Medical Research Council of streptomycin in tuberculosis.

\title{
NON-TUBERCULOUS MENINGITIS
}

\author{
By George Newns, M.D., M.R.C.P. \\ Physician to the Hospital for Sick Children, Great Ormond Street
}

\section{(1) PURULENT MENINGITIS}

The treatment of purulent meningitis has undergone a great change in the past ten years. Before the introduction of sulphonamide therapy, the prognosis in all but meningococcal meningitis was very bad and there were very few recoveries. A radical transformation has been brought about by modern chemotherapy and even cases of influenzal meningitis with early and thorough treatment, may now be expected to recover, although hitherto they were almost always fatal. Nevertheless many patients with purulent meningitis still die, due largely to failure to diagnose the disease early enough and to inadequate treatment.

Diagnosis. The diagnosis of acute meningitis is not difficult although in infants the onset may be more insidious and typical meningeal signs absent. As a rule the onset is very acute, sudden high fever developing in a previously healthy child; drowsiness is an early symptom. Pain in the neck muscles especially on flexion soon appears and Kernig' and Brudzinski's signs become positive.

If the child is untreated he becomes stuporose with marked neck retraction and in severe cases opisthotonus may develop. Vomiting follows as a result of rise in the intra-cranial pressure (in infants under two years the sutures may separate and hydrocephalus may develop).

It is not easy to distinguish one form of purulent meningitis from another, but if purpura and small haemorrhagic spots appear, the infection is probably due to the meningococcus. In infants, acute meningitis often does not present the typical syndrome outlined above. Neck stiffness may be absent, and Kernig's sign is often negative. Irritability, drowsiness, photophobia, bulging of the fontanelle in a child with unexplained fever are very suggestive of the onset of meningitis and a lumbar puncture should be carried out without delay.

DIFFERENTIAL DIAGNOSIS OF MENINGITIS

\begin{tabular}{|c|c|c|c|c|c|c|c|c|}
\hline \multirow{2}{*}{\multicolumn{2}{|c|}{ Tuberculous Meningitis .. }} & \multirow[b]{2}{*}{. } & \multirow[b]{2}{*}{. } & Polymorphs & Mononuclears & Sugar & Chlorides & Protein \\
\hline & & & & & + & Decreased & Very low & + \\
\hline Purulent Meningitis & . & $\ldots$ & . & +++ & & Absent & Slightly decreased & + \\
\hline Virus Meningitis . . & . & . & $\ldots$ & & + & Normal & Normal & + \\
\hline Serous Meningitis & . & . & . & + & & Normal & Normal & + \\
\hline
\end{tabular}


A diagnosis of the type of meningitis should be made as early as possible in order that the proper treatment may be given. A diagnosis can be arrived at in meningitis due to the pneumococcus, $H$. influenzae and the meningococcus, within an hour, by the use of stained smears and type specific anti-serum. By estimating the glucose in the C.S.F. quantitatively, the severity of the infection can be assessed. The lower the level of glucose the more severe is the infection.

\section{Meningococcal Meningitis}

This disease takes several forms. The meningitic variety presents symptoms indistinguishable from other types of purulent meningitis, except that haemorrhagic skin lesions are common. Neck stiffness and head retraction are often marked and opisthotonus may occur. The fulminating type runs a rapid course and coma supervenes early. Extensive purpura is present and there are frequently massive haemorrhages into the suprarenals (Waterhouse-Friderichsen syndrome). In a less acute variety, symptoms of septicaemia may precede the onset of meningitis by several days. Mild forms occur especially in infants and may be difficult to recognize from the clinical symptoms, as signs of meningitis are absent or inconspicuous.

The chronic type of meningitis succeeds the ordinary acute attack ; it is especially frequent in infants. Pathologically there is a persistent basal exudate which causes obstructive hydrocephalus. Frederic Still was the first to prove that chronic, basic meningitis was due to infection by the meningococcus. This type may last for several months, and is, as a rule, ultimately fatal.

Treatment by anti-serum was effective in a considerable proportion (about 70 per cent.) of cases of meningococcal meningitis before the introduction of modern chemotherapy. Some physicians still use serum, but this would now seem to be unnecessary in view of the efficacy of sulphonamides and penicillin.

Penicillin by itself is not as effective as the sulphonamides. Most cases, if treated within 48 hours of onset, react well to sulphonamide therapy, and penicillin is indicated only when there is no response to sulphonamides after 48 hours' treatment. Sulphadiazine is the sulphonamide of choice and it should be administered in sufficient dosage to secure a blood level of 8 to $12 \mathrm{mg}$. per roo cc. An initial dose of 0.1 G. per pound body weight is given intramuscularly, followed by the same dose by mouth each day, divided into 4-6 doses. In severe cases the initial dose may be given intravenously. If the patient is unable to swallow, subsequent doses may have to be administered by intramuscular injection, or by stomach tube. It is important to keep up the fluid intake and fluids may be given parenterally or by stomach tube.

Frequent lumbar punctures are unnecessary. An initial, diagnostic one is required, another after three days and another at the end of about a week or ten days to determine the effect of treatment. The drug may be discontinued after the temperature has been normal for two days. Penicillin should be employed only as an adjuvent to sulphonamide therapy. It should be used in cases of fulminating meningococcal infections from the onset of treatment. In the ordinary variety it should be employed only if there is no response to sulphonamides after 48 hours.

The drug should be given intrathecally in doses of 10,000-20,000 units, once or twice daily, according to the severity of the infection. The white crystalline penicillin should be used, as those penicillins containing impurities cause irritation of the meninges.

In the fulminating cases, when there is reason to suspect suprarenal haemorrhage, adrenocortical extract should be given. These patients are very shocked and exhibit peripheral circulatory collapse ; intravenous injections of fluid, saline and plasma are indicated. The penicillin can then be given by the intravenous route.

\section{Influenzal Meningitis}

Infection of the meninges by $\mathrm{H}$. Influenzae is by no means a rare condition. Four cases have come under my personal care in the past year in one hospital alone. Of these, three recovered completely without sequelae, and the remaining one recovered but developed hydrocephalus and is blind.* All had combined sulphonamide and streptomycin therapy.

The one that had sequelae however was not admitted to hospital until eight days after the onset of the illness. Up to ten years ago, the mortality of influenzal meningitis was nearly roo per cent.

The meningitis has usually a sudden onset and may follow an upper respiratory tract infection (in one of the above cases it was preceded by a pneumonia). It is encountered mainly in infancy, but it does not occur in the newborn, who are protected by antibodies derived from the mother.

The organisms are present in large numbers in the C.S.F. and are often pleomorphic in appearance. They are easily cultured. All cases of influenzal meningitis are caused by the serologically related strains called type $b$. It is now possible to prepare an anti-body against this type b strain. Hattie Alexander has prepared a very potent antiserum from rabbits which is now used in the

*These cases were treated at the London Hospital. 
treatment of the disease. According to Alexander the ideal treatment is a combination of sulphadiazine and rabbit type specific anti-serum. Sulphadiazine appears to be the most effective of the sulphonamides. Rabbit serum is unobtainable in this country and streptomycin has been used with very good results in combination with sulphadiazine.

Management of case. Early treatment is most essential. If treatment is delayed the disease may become chronic and loculations of pus develop in the meninges (especially over the base of the brain) and the infant then becomes resistant to further treatment. Hydrocephalus, cranial nerve palsies, etc., result.

Alexander first administers a subcutaneous dose of sulphadiazine ( $0.05 \mathrm{G}$. per lb. body weight). In fulminating infections an intravenous infusion of sixth-molar sodium lactate solution, in 5 per cent. glucose may be given and the sulphadiazine added to the infusion fluid. In milder attacks, or those recovering sufficiently, fluids are given by mouth, providing no vomiting occurs.

To the infusion reservoir, the calculated dose of anti-body, diluted with 5 cc. of isotonic sodium chloride per $\mathrm{lb}$. body weight is added and given at such a rate that the whole is given in a period of two hours. Otherwise the anti-serum is given directly into a vein. The dose of anti-body is determined by the level of the sugar in the C.S.F.; if there is less than $15 \mathrm{mg}$. per I0O cc., I00 $\mathrm{mg}$. of anti-body nitrogen is given; if over $40 \mathrm{mg}$., 25 mg. of anti-body is injected. Further doses of antibody, given intravenously, depend on the level of the C.S.F. glucose and the capsular-swelling power of the patient's serum, which is tested daily. The subcutaneous dose of sulphadiazine is repeated at 12-hourly intervals, if the patient is unable to swallow. Subsequent doses may be given by mouth (o.I G. per lb.) in six divided doses daily, i.e. at four-hourly intervals. It is essential to keep up an adequate fluid intake either by mouth or parenterally.

Those patients in whom the sugar content of the C.S.F. is $40 \mathrm{mgm}$. or higher, and in whom treatment is known to have started within 24 hours of the commencement of the illness, may be treated successfully by sulphadiazine alone, provided sufficient amounts are given to maintain a blood level of 10-1 $5 \mathrm{mg}$. per cent. The sulphadiazine, if used alone, should be continued for two weeks after the C.S.F. has become sterile; if combined with the anti-body one week is sufficient.

Streptomycin has recently been employed effectively in the treatment of influenzal meningitis. Most strains of the organism are very sensitive in vitro to the organisms. The drug is administered intramuscularly (100-1 $50 \mathrm{mg}$. each six hours), but it is also necessary to supplement with daily intrathecal injections $(30-50 \mathrm{mg}$.) as adequate levels in the C.S.F. cannot be attained by intramuscular injections alone. The drug should not be given for longer than a week as cases of eighth nerve deafness have followed prolonged treatment. It should be combined with sulphadiazine therapy to obtain the best results. Streptomycin, plus sulphadiazine is effective even in severe cases, provided treatment is started early.

Although $\mathrm{H}$. Influenzae is generally resistant to penicillin, some strains have been found to be sensitive. Several such cases of $\mathrm{H}$. Influenzae meningitis have been treated recently by penicillin, with success at the Hospital for Sick Children, Great Ormond Street.

To summarize treatment :-

Mild cases, if treated early, can be cured by sulphadiazine, streptomycin or rabbit-type specific anti-body used singly. More severe cases require a combination of therapeutic agents, either sulphadiazine and streptomycin, or sulphadiazine and rabbit anti-serum. Occasionally all three are indicated.

\section{Pneumococcal Meningitis}

This disease has always had .a high mortalit and even today is often fatal. The disease is particularly severe in infants, loculation is liable to occur and the infection may become chronic. Early treatment is vital. Treatment with sulphonamides alone is disappointing. In Dingle and Finland's review (1942), 3 I to 80 per cent. died.

As soon as the diagnosis is made, an injection of soluble sulphadiazine should be given in a dosage of $0.05 \mathrm{G}$. per lb. body weight. Oral administration is started at the same time, an initial dose of $0.05 \mathrm{G}$. per lb. of body weight being given (by mouth or stomach tube). Subsequently 0.I G. per lb. of body weight is given every 24 hours in divided doses. A blood concentration of 8-12 mg. per roo cc. should be attained.

Penicillin is very effective in pneumococcal meningitis, in combination with sulphonamide therapy. Large doses should be given intramuscularly. For several days a daily dose of 10,000 to 20,000 units should be administered intrathecally, as only a small proportion diffuses into the C.S.F. A long period of treatment may be necessary to prevent relapse. In America, type specific rabbit anti-serum has been given as soon as the type of pneumococcus has been determined. (It is combined with sulphadiazine or penicillin, or both.) Indeed in very young infants, in whom the disease is very severe, one probably requires all three agents to effect a cure. 


\section{Streptococcal Meningitis}

This is a fairly common type of meningitis and is especially frequent in infancy. It may follow a septicaemia. In infancy it may result from an umbilical infection and in older children it may be secondary to otitis media and mastoiditis. The most effective treatment is a combination of sulphonamides and penicillin.

The former should be given in doses sufficient to produce a blood level of 8-I2 mg. per Ioo cc. Penicillin should be administered intramuscularly at frequent intervals, and by the intrathecal route, in doses of $10,000-20,000$ units once or twice daily.

\section{Staphylococcal Meningitis}

This is a relatively uncommon condition. It is usually a haematogenous infection from a primary focus, such as a cutaneous infection, mastoiditis, etc. The disease is a serious one, and until recently the mortality was high. Abscess formation is common.

The sensitivity of the strain of staphylococcus to penicillin should be determined as soon as it is isolated and the dose of the drug adjusted accordingly. The drug should be administered in optimum dosage for at least one week after the C.S.F. has become sterile.

\section{Bact. Coli. Meningitis}

This is a fairly common form of meningitis in young infants. There is in most cases a bacteraemia. The sulphonamides in adequate doses are an effective treatment. In rẹsistant cases, however, streptomycin should be employed as the drug has a marked bactericidal effect on all the colon group of organisms.

\section{(2) NON-PURULENT MENINGITIS}

There occur several types of meningitis in which the C.S.F. is clear and in which lymphocyte cells predominate.

\section{Lymphocytic Chorio-Meningitis}

This is an acute virus infection of the central nervous system; the virus is found in mice and monkeys, as well as in man. Mice appear to carry the virus without exhibiting symptoms and it is considered that they may be a possible source of human infection. The virus is easily isolated from the blood and C.S.F. of patients suffering from the disease, and when injected into guinea pigs will cause death in about two weeks.

The symptoms are sudden onset with fever, headache, vomiting, stiff neck and usually a positive Kernig's sign. The C.S.F. is under pressure and the cells are increased to a varying extent, from 200 to as many as 2,000 in some cases.
Over 80 per cent. are lymphocytes. The protein is slightly raised, but the sugar content is normal. The duration of the illness is on the average ro to I4 days, but in some instances it may last considerably longer.

Diagnosis often presents difficulties. Tuberculous meningitis has to be excluded. The acute onset is in favour of lymphocytic meningitis and in the C.S.F. the normal sugar and chloride levels are against tuberculous meningitis, although in the early stages of this disease these may be little changed. Careful search of the C.S.F. in the latter infection generally reveals tubercle bacilli. The progress of the case will usually clear up any doubts as to diagnosis. Since the introduction of streptomycin, however, it is wise in all doubtful cases to treat as for a case of tuberculous meningitis and commence streptomycin therapy while awaiting the result of culture of the C.S.F.

Lymphocytic meningitis may be very difficult to distinguish from early acute anterior poliomyelitis when there are meningitic symptoms. The C.S.F. in this disease may closely resemble that of lymphocytic meningitis and it may be impossible to make a diagnosis at the outset of the illness.

There is no specific treatment for lymphocytic chorio-meningitis. Recovery is spontaneous and there appear to be no complications or sequelae.

\section{Mumps-Meningo-Encephalitis}

A meningitis is not infrequently a complication of mumps and may occur in the absence of a parotitis; it is not a pure meningitis, but is associated with an encephalitis; meningo-encephalitis is therefore a better term. In some cases the meningitis, and in other cases the encephalitis is the predominant feature of the infection. The symptoms are acute, with rapid onset of meningeal symptoms; if the encephalitis is severe, convulsions may occur.

The C.S.F. is clear ; there is a variable increase in the lymphocytes, which may be as high as $\mathrm{I}, 000$ per c.cm., but there is no change in the sugar and chloride level, although the protein is usually slightly raised. Death may occur when the encephalitis is severe but recovery is the rule and is usually complete

In glandular fever there may be central nervous system complications, and in recent years there have been a number of reports of meningitis, headache and neck stiffness; mental confusion may arise in the course of the disease and on examination of the C.S.F. there is an increase in the lymphocytes and some increase in the protein content. The prognosis is good and recovery takes place without sequelae. 


\section{Serous Meningitis or Aseptic Meningitis}

This is the name given to the syndrome in which a meningeal reaction occurs as the result of an infective focus adjacent to the meninges, e.g. mastoiditis, lateral sinus thrombosis, extradural abscess, intra-cerebral abscess, etc. The pressure of the C.S.F. is increased, and there is an increase in cells, generally polymorphs, but sometimes lymphocytes predominate. The protein is slightly increased, but the sugar and chlorides are normal. The cell count is usually in the neighbourhood of $200-300$, but occasionally it is as high as 1,000 per c.cm. Organisms are not present. In some cases an actual meningitis may supervene.

A lymphocytic meningitis is not an infrequent occurrence in the early stages of Weil's disease and an example came under my own observation recently. The lymphocytes were several hundreds per c.cm. and took two-three weeks to return to normal. There was no jaundice in this patient and the diagnosis was made by finding a high agglutination titre in the blood.

\section{BIBLIOGRAPHY}

ALEXANDER, H. E., 'Treatment of Haemophilus Influenzae Infections, and of Meningococci and Pneumococci Meningitis,' Amer. Four. Dis. Child. Aug., r 943, lxvi, 160.

DINGLE, J. N., FINLAND, M. (1942), War Med., 2, I.

\title{
MENINGITIS: RATIONALE OF DIAGNOSIS
}

\author{
By. JohN APLEY, M.D., M.R.C.P. \\ Department of Child Health, Bristol University
}

Increased power entails increased responsibility. With the advent of chemotherapy delay in the diagnosis of purulent meningitis can no longer be excused, for diagnosis at leisure is condonable only when dealing with an incurable or benign disease. Virtually all the common varieties of purulent meningitis are now curable, yet even now many patients die, or survive only with distressing residua. Inadequacy of laboratory facilities and improper selection of therapeutic agents are contributory factors in the production of these failures ; but the most important single factor is delay in diagnosis.

It is axiomatic in medicine that urgent diagnosis depends on leisurely preparation. The diagnostician who has failed to discipline himself by repeated observation, analysis and synthesis will fail also to attain that maturity of judgment which permits diagnosis to be made rapidly yet without the sacrifice of accuracy. The study of meningitis provides an example of the preliminary, catholic preparation which is essential for urgent diagnosis. Though the present discussion is centred on purulent meningitis the logical approach must be through the subject of lepto-meningitis in its wider aspects.

\section{Features of Meningeal Irritation}

The essential feature common to the many forms of meningitis is meningeal irritation. This phenomenon may occur even in the absence of infection of the meninges; but, whatever the cause of the irritation, the clinical features which may be observed result in all cases from stimula- tion of the meningeal nerves and those portions of the cranial and spinal nerves which traverse the theca. The mechanism is identical with that obtaining in peritonitis, and the results are similar.

The three characteristic features of meningeal irritation are :-(I) pain, (2) hyperaesthesia, and (3) muscular rigidity. The first two of these arise from irritation of sensory components, and the third through the mechanism of a meningealmotor reflex.

The various signs, such as those of Kernig and Brudzinski, and the demonstration of neck rigidity, which are employed in the diagnosis of meningitis, depend for their effects upon increasing the irritability of the meninges by mechanical stretching.

Correlation of Symptomatology with Site of Meningeal Irritation

The production of the three characteristic features of meningeal irritation can be clearly illustrated by contrasting anterior poliomyelitis with meningococcal meningitis. At the same time the discussion can be taken a stage further to provide evidence on which to attempt localization of the site of meningeal involvement.

In anterior poliomyelitis, when the site of predilection is in the lumbar region of the cord, the manifestations of meningeal irritation appear first in the back and the legs; from these regions they may spread or remain localized, according as the disease process spreads or is halted. In meningococcal meningitis, when, as commonly occurs, the site of predilection is at the base of the brain, the 